\title{
HOMOGENEOUS FOLIATIONS OF SPHERES
}

\author{
DUOJIA LU
}

\begin{abstract}
In this paper we discuss Riemannian foliations of the round sphere. We prove that there are no homogeneous Riemannian foliations of the round sphere with dimensions of the leaves bigger than three.
\end{abstract}

\section{Definitions and the statement of the Main Theorem}

A foliation $\left(M^{n}, \mathscr{F}\right)$ of an $n$-dimensional smooth manifold $M^{n}$ is a $k$ dimensional integrable subbundle of the tangent bundle $T M^{n}$ of $M^{n}(k \leq$ $n$ ). The maximal integral submanifolds of dimension $k$ are the leaves of the foliation $\left(M^{n}, \mathscr{F}\right)$. We are only concerned with nontrivial foliations: $0<k<$ $n$.

Let $\left(M^{n}, g\right)$ be a connected $n$-dimensional Riemannian manifold with the metric $g$. Then a foliation $\left(M^{n}, \mathscr{F}\right)$ is called a Riemannian foliation if the leaves are locally equidistant, namely, there is a small ball around every point of $M^{n}$ such that the intersections of the leaves with the ball are parallel. Locally the leaves are the fibers of a Riemannian submersion.

Let $G$ be a Lie subgroup of Iso $\left(M^{n}, g\right)$. Without loss of generality, we may assume that $G$ is connected. Then the orbits of $G$ are locally equidistant. If, furthermore, the orbits of $G$ are of the same dimension through every point, we have the important case of a homogeneous Riemannian foliation. For the round sphere $S^{n}$, Gromoll and Grove proved the following theorem [6, 7].

Theorem 1.1 (Gromoll-Grove). Let $\left(S^{n}, \mathscr{F}\right)$ be a Riemannian foliation of the round sphere $S^{n}$. Then

(1) If $\mathscr{F}$ has 1 -dimensional fibers, then $\mathscr{F}$ is a homogeneous Riemannian foliation given by the orthogonal action of $\mathbf{R}^{1}$ on $\mathbf{C}^{k}=\mathbf{R}^{2 k} \quad(n=2 k-1)$, which can be described after conjugation as

$$
\begin{aligned}
e^{2 \pi i t}\left(z_{1}, z_{2}, \ldots, z_{n}\right)=\left(e^{2 \pi i \theta_{1} t} z_{1}, e^{2 \pi i \theta_{2} t} z_{2}, \ldots, e^{2 \pi i \theta_{k} t} z_{k}\right), \\
0<\theta_{1} \leq \theta_{2} \leq \cdots \leq \theta_{k} \leq 1 .
\end{aligned}
$$

These foliations are Seifert fibrations (i.e., all fibers are compact) iff all $\theta_{i}$ 's are rational. There is only one filtration among these foliations, which is the Hopf fibration $S^{1} \rightarrow S^{2 k-1} \rightarrow \mathbf{C} P^{k-1}$ corresponding to $\theta_{1}=\theta_{2}=\cdots=\theta_{k}=1$.

(2) $\mathscr{F}$ cannot have 2 -dimensional fibers.

Received by the editors October 9, 1990.

1980 Mathematics Subject Classification (1985 Revision). Primary 53C12.

(C) 1993 American Mathematical Society $0002-9947 / 93 \$ 1.00+\$ .25$ per page 
(3) If $\mathscr{F}$ has 3-dimensional fibers, then $\mathscr{F}$ is a homogeneous Riemannian foliation given by the direct sum of orthogonal irreducible representations of $\mathrm{SU}(2)$ on $\mathbf{C}^{2 k}=\mathbf{R}^{4 k}$, which can be described after conjugation as

$$
V_{m_{1}} \oplus V_{m_{2}} \oplus \cdots \oplus V_{m_{j}}, \quad 1 \leq m_{1} \leq m_{2} \leq \cdots \leq m_{j},
$$

where the $m_{i}$ 's are odd positive integers and $V_{m_{i}}$ is the irreducible representation of $\mathrm{SU}(2)$ on the complex space of homogeneous polynomials in two variables of degree $m_{i}$. These foliations are all Seifert fibrations. The only fibration among these is the Hopf fibration $S^{3} \rightarrow S^{4 k-1} \rightarrow \mathbf{H} p^{k-1}$ corresponding to $m_{1}=m_{2}=$ $\cdots=m_{j}=1$.

According to the above theorem of Gromoll and Grove, all the low dimensional Riemannian foliations of the round spheres are homogeneous. In this paper, we prove the following result which classifies the homogeneous Riemannian foliations of the round spheres.

Main Theorem 1.2. If $\left(S^{n}, \mathscr{F}\right)$ is a nontrivial homogeneous Riemannian foliation on the round sphere $S^{n}$, then $\mathscr{F}$ has either 1- or 3-dimensional fibers, and $\mathscr{F}$ can be described as in Theorem 1.1.

Remark. Once one knows that the dimensions of the fibers of $\mathscr{F}$ are either one or three, one can argue directly as in the following proof or one can use the statement of Theorem 1.1 to get the description of $\mathscr{F}$.

The classification of the Riemannian foliations of the round spheres is related to the following rigidity theorem of Gromoll and Grove [5].

Theorem 1. 3 (Gromoll-Grove). Let $M$ be a complete Riemannian manifold of dimension $n \geq 2$ with sectional curvature $K \geq 1$ and $\operatorname{diam}(M)=\pi / 2$, then $M$ is a homotopy sphere or $\widetilde{M}$, the universal cover of $M$, is isometric to a rank-one symmetric space, except possibly when $M$ has the integral cohomology ring of the C'ayley projective plane $\mathrm{Ca} P^{2}$.

In the proof of this theorem, Gromoll and Grove applied their result on the low dimensional Riemannian foliations of the round sphere $S^{n}$. Correspondingly, if one is able to show the uniqueness of the Riemannian foliations of $S^{n}$ with fibers which are homotopy seven-spheres, then one can improve the above rigidity theorem of Gromoll and Grove to say that either $M$ is a homotopy sphere or $\widetilde{M}$ is isometric to a rank-one symmetric space.

\section{Proof of the Main Theorem}

The idea of the proof of the Main Theorem 1.2 is to determine all equivalence classes of orthogonal actions of connected Lie groups on $S^{n}$ whose isotropy group at every point has the same dimension. Hence we look at all linear representations of a compact connected Lie group and compute the dimensions of the isotropy groups at each point of $S^{n}$. For convenience, we assume that all representations are faithful.

We achieve the proof of the Main Theorem 1.2 in three steps.

Step I. We prove the following proposition which enables us to consider only the irreducible representations. 
Proposition 2.1. Let $\Phi: G \rightarrow \mathrm{SO}(n+1)$ be a faithful representation of a connected Lie group $G$ of rank larger than one. If $\Phi$ gives rise to a homogeneous foliation, then $\Phi$ is irreducible.

Proof. Assume $\Phi$ is not irreducible, i.e., after conjugation, we have the complete, irreducible decomposition of $\Phi$ into $\Phi=\Phi_{1} \oplus \Phi_{2} \oplus \cdots \oplus \Phi_{k}$, where $\Phi_{j}: G \rightarrow \mathbf{S O}\left(i_{j}\right), j=1,2, \ldots, k$, and $n+1=i_{1}+i_{2}+\cdots+i_{k}$. Hence for $g \in$ $G$, and $x=\left(x_{1}, x_{2}, \ldots, x_{k}\right), \Phi(g) x=x$ iff $\Phi_{j}(g) x_{j}=x_{j}, j=1,2, \ldots, k$. It follows that if $G_{x}^{0}$ and $G_{x_{j}}^{0}(j=1,2, \ldots, k)$ denote the identity components of the isotropy groups $G_{x}$ at $x$ and $G_{x_{j}}$ at $x_{j}$ for $j=1,2, \ldots, k$, then $G_{x}^{0} \subseteq \bigcap\left\{G_{x_{j}}^{0}: x_{j} \neq 0,1 \leq j \leq k\right\}$. Since $G_{x}^{0}$ and $G_{x_{j}}^{0}$ are of the same dimension, we have $G_{x}^{0}=G_{x_{j}}^{0}$ if $x_{j} \neq 0 \quad(1 \leq j \leq k)$. This implies that $G_{x}^{0}$ is a fixed subgroup of $G$ for all $x \in G$. Since $\Phi$ is faithful, we have $G_{x}^{0}=\{e\}$, where $e$ is the identity element of $G$. This means that every isotropy subgroup of $G$ is discrete. Therefore a maximal torus of $G$ acts on $S^{n}$ locally freely. By diagonalization we see that this action must be a circle action. Hence $G$ is a rank-one Lie group. Q.E.D.

Corollary 2.2. If a homogeneous Riemannian foliation $\mathscr{F}$ is given by a reducible representation, then $\mathscr{F}$ has 1- or 3-dimensional fibers.

Proof. By Proposition $2.1 G$ is isomorphic to $S^{1}, \mathbf{R}^{1}, \mathrm{SU}(2)$, or $\mathrm{SO}(3)$ and the action is free by using the same argument as in the proof of Proposition 2.1. Clearly all the locally free orthogonal actions of any rank-one connected Lie group give rise to homogeneous Riemannian foliations with 1 - or 3- dimensional fibers. Q.E.D.

Remark. One can show that if the action of a group $G$ gives rise to a nonsingular orbit foliation of a manifold, then the rank of the isotropy groups is equal to that of $G$ minus one.

Therefore, we only need to consider the irreducible representations.

Step II. Now we prove the following proposition which allows us to consider only the case where $n=15$ and $k=7$.

Proposition 2.3. Let $\mathscr{F}$ be a Riemannian foliation of the round sphere $S^{n}$ with $k$-dimensional fibers. Then $k=1,3$, or 7 . When $k=14$, then $n=2 m+1$ $(m=1,2, \ldots)$; when $k=3$, then $n=4 m+3(m=1,2, \ldots)$; and when $k=7$, then $n=15$.

The proof of this proposition is a direct application of several known theorems. Here we list the theorems which will be used without proof.

Theorem 2.4 (Ghys [4]). Let $S$ be a simply-connected Riemannian manifold which is a rational homology sphere. If $\mathscr{F}$ is a Riemannian foliation on $S$, then $\mathscr{F}$ is either a Seifert fibration or $\mathscr{F}$ has 1-dimensional fibers.

Theorem 2.5 (Haefliger [8]). Let $\mathscr{F}$ be a Seifert fibration on a compact manifold $M$. Let $F$ be the generic fiber of $\mathscr{F}$ and $X$ be the space of leaves. Then there is a space $B X$ and a continuous map from $M$ onto $B X$ which is homotopically equivalent to a locally trivial fibration with base $B X$ and fiber $F$. Furthermore, there exists a natural map from $B X$ to $X$ inducing an isomorphism in rational cohomology. 
Theorem 2.6 (Browder [3]). If $p: S^{2} \rightarrow B$ is a fiber map, with base $B$ and fiber $F$ connected polyhedra, $B \neq$ point, then $F \cong S^{1}, S^{3}$ or $S^{7}$. In case $F \cong S^{7}$, $B \cong S^{8}$.

Proof of Proposition 2.3. Suppose $k=1$, then $\mathscr{F}$ is given by a representation of $\mathbf{R}^{1}$ on $\mathbf{C}^{m+1}$ by Theorem 1.1. Suppose $k>1$, by Theorem $2.4, \mathscr{F}$ is a Seifert fibration. Then Theorems 2.5 and 2.6 imply that $k=3$ or $k=7$, and when $k=7$, then $n=15$. When $k=3$, an easy computation of cohomology shows that $n=4 m+3$. Q.E.D.

Remark. In case that $\mathscr{F}$ is a homogeneous foliation, the generic fibers are simply the principal orbits of the group action by $G$. Hence the generic fiber is a homogeneous manifold which is also a homotopy sphere. It is well known that if a homogeneous manifold is a homotopy sphere, then it is diffeomorphic to the standard sphere (Besse [1]). Therefore the generic fibers are in fact diffeomorphic to spheres.

Step III. Now we have to look at irreducible orthogonal actions of connected Lie groups on $S^{15}$ and show that none of these groups has principal orbit diffeomorphic to the sphere $S^{7}$. The following is a complete list of pairs $(G, H)$ of connected Lie groups such that $G / H$ is diffeomorphic to $S^{7}$ (Besse [1]).

(1) $(\mathrm{SO}(8), \mathrm{SO}(7))$.

(2) $(\mathrm{SU}(4), \mathrm{SU}(3))$.

(3) $(\mathrm{U}(4), \mathrm{U}(3))$.

(4) $(\mathrm{Sp}(2), \mathrm{Sp}(1))$.

(5) $(\mathrm{Sp}(2), \mathrm{U}(1), \mathrm{Sp}(1) \mathrm{U}(1))$.

(6) $(\mathrm{Sp}(2) \mathrm{Sp}(1), \mathrm{Sp}(1) \mathrm{Sp}(1))$.

(7) $\left(\operatorname{Spin}(7), G_{2}\right)$.

Therefore, for each couple in the above list, we need to look at the faithful irreducible representations of the group $G$ of proper dimensions, namely those representations which give us orthogonal actions of $G$ on $S^{15}$ irreducibly and faithfully. For short, we call these representations admissible.

We denote the equivalence classes of complex irreducible representations of real type, complex type, and quaternionic type by $\operatorname{Irr}(G, \mathbf{C})_{\mathbf{R}}, \operatorname{Irr}(G, \mathbf{C})_{\mathbf{C}}$, and $\operatorname{Irr}(G, \mathbf{C})_{\mathbf{H}}$ respectively. Similarly, we denote the equivalence classes of real irreducible representations of real, complex, and quaternionic types by $\operatorname{Irr}(G, \mathbf{R})_{\mathbf{R}}, \operatorname{Irr}(G, \mathbf{R})_{\mathbf{C}}$ and $\operatorname{Irr}(G, \mathbf{R})_{\mathbf{H}}$, respectively. For a complex representation $V$, we let $r_{\mathbf{R}}^{\mathrm{C}} V$ be the underlying real representation. It is well known that if $V \in \operatorname{Irr}(G, \mathbf{C})_{\mathbf{R}}$, then $r_{\mathbf{R}}^{\mathbf{C}} V=\mathrm{U} \oplus \mathrm{U}$ where $\mathrm{U} \in \operatorname{Irr}(G, \mathbf{R})_{\mathbf{R}}$. If $V \in \operatorname{Irr}(G, \mathbf{C})_{\mathbf{C}}$, then $r_{\mathbf{R}}^{\mathbf{C}} V \in \operatorname{Irr}(G, \mathbf{R})_{\mathbf{C}}$, and if $V \in \operatorname{Irr}(G, \mathbf{C})_{\mathbf{H}}$, then $r_{\mathbf{R}}^{\mathrm{C}} V \in \operatorname{Irr}(G, \mathbf{R})_{\mathbf{H}}$ (see [2]). Hence the admissible representations come from the complex 16-dimensional irreducible representations of real type, and the complex 8-dimensional irreducible representations of complex of quaternionic types.

We can derive the possible dimensions of admissible representations for $G$ of all couples in the above list by computations using Weyl's formula. The results are as follows:

(1) $\mathrm{SO}(8)$. For $\mathrm{SO}(8)$, there are three complex 8-dimensional irreducible representations and no 16-dimensional ones. Since all complex irreducible rep- 
resentations of $\mathrm{SO}(2 k)$ for $k$ even are self-conjugate and of real type, these representations are not admissible.

(2) $\mathrm{SU}(4)$. For $\mathrm{SU}(4)$, there are no irreducible complex representations of eight and sixteen dimensions.

(3) $U(4)$. Since $S U(4) \times U(1) \rightarrow U(4)$ is a covering map, any irreducible representation of $U(4)$ lifts up to an irreducible representation of $S U(4) \times U(1)$. On the other hand, any irreducible representation of $S U(4) \times U(1)$ is of the form $V \otimes V^{\prime}$, where $V$ and $V^{\prime}$ are irreducible representations of $\mathrm{SU}(4)$ and $\mathrm{U}(1)$ respectively. But $V^{\prime}$ is 1 -dimensional since $\mathrm{U}(1)$ is abelian. Hence the dimension of $V \otimes V^{\prime}$ is the same as that of $V$. It follows that there are no irreducible complex representations of dimensions eight and sixteen for $U(4)$ from the previous argument.

(4) $\mathrm{Sp}(2)$. For $\mathrm{Sp}(2)$, there is only one complex irreducible representation of possible dimension sixteen. This representation has the highest weight $\lambda=\lambda_{1}+\lambda_{2}$, where $\lambda_{1}$ and $\lambda_{2}$ are the fundamental weights. Let $V_{1}$ and $V_{2}$ be the irreducible representations of $\mathrm{Sp}(2)$ corresponding to the fundamental weights $\lambda_{1}$ and $\lambda_{2}$. Then the irreducible representation corresponding to $\lambda$ is the Cartan composite $V=V_{1} * V_{2}$, which is the irreducible summand of multiplicity one in $V_{1} \otimes V_{2}$ with the highest weight $\lambda=\lambda_{1}+\lambda_{2}$. The irreducible representation $V_{1}$ of the fundamental weight $\lambda_{1}$ is the natural action of $\operatorname{Sp}(2)$ on $\mathbf{H}^{2} \cong \mathbf{C}^{4}$. The fundamental representation $V_{2}$ is 5 -dimensional and real, which actually can be identified as the natural action of $\mathrm{SO}(5)$ on $\mathbf{C} \otimes \mathbf{R}^{5}$. Therefore, $V$ is of quaternionic type which is not admissible.

(5) $\mathrm{Sp}(2) \mathrm{U}(1)$. The irreducible representations of $\operatorname{Sp}(2) \mathrm{U}(1)$ are of the form $V \otimes V^{\prime}$, where $V$ is an irreducible representation of $\operatorname{Sp}(2)$ and $V^{\prime}$ of $\mathrm{U}(1)$. The fact that $\mathrm{U}(1)$ is abelian implies that $V^{\prime}$ is 1-dimensional. So $V \otimes V^{\prime}$ has the same dimension as that of $V$. From (4), we know that the only possible dimension for admissible representation of $\mathrm{Sp}(2) \mathrm{U}(1)$ is sixteen, and $V$ is of quaternionic type. Clearly, $V^{\prime}$ is not self-conjugate. Hence $V$ and $V^{\prime}$ are not of the same type. Since $V \otimes V^{\prime}$ is self-conjugate iff $V$ and $V^{\prime}$ are of the same type, then $V \otimes V^{\prime}$ is not self-conjugate, therefore not of real type. This implies there are no admissible representations for $\operatorname{Sp}(2) \mathrm{U}(1)$.

(6) $\mathrm{Sp}(2) \mathrm{Sp}(1)$. Let $V \otimes V^{\prime}$ be an irreducible representation of $\mathrm{Sp}(2) \mathrm{Sp}(1)$, where $V$ is any irreducible representation of $\operatorname{Sp}(2)$ with the highest weight $\lambda=m_{1} \lambda_{1}+m_{2} \lambda_{2} . m_{1}, m_{2} \in \mathbf{Z}_{+}, \lambda_{1}$ and $\lambda_{2}$ the fundamental weights, and $V^{\prime}$ is any irreducible representation of $\mathrm{Sp}(1)$ with the highest weight $m \mu$, $m \in \mathbf{Z}_{+}, \mu$ the fundamental weight. By Weyl's formula, we see that there are two complex 8-dimensional irreducible representations of $\mathrm{Sp}(2) \mathrm{Sp}(1)$ corresponding to

(i) $\left(m_{1}, m_{2}, m\right)=(0,0,7)$,

(ii) $\left(m_{1}, m_{2}, m\right)=(1,0,1)$.

There are also three complex 16-dimensional irreducible representations corresponding to

(iii) $\left(m_{1}, m_{2}, m\right)=(0,0,15)$,

(iv) $\left(m_{1}, m_{2}, m\right)=(1,1,0)$,

(v) $\left(m_{1}, m_{2}, m\right)=(1,0,3)$.

In cases (i), (iii), and (iv), either $\operatorname{Sp}(2)$ or $\mathrm{Sp}(1)$ acts trivially, therefore the representations are not admissible. 
In case (ii), the corresponding representation is $V \otimes V^{\prime}$, where $V$ is the fundamental representation of $\operatorname{Sp}(2)$ with the highest weight $\lambda=\lambda_{1}$, and $V^{\prime}$ is the fundamental representation of $\operatorname{Sp}(1)$ with the highest weight $\mu$. In fact $V$ is the standard representation of $\operatorname{Sp}(2)$ on $\mathbf{H}^{2} \cong \mathrm{C}^{4}$, and $V^{\prime}$ is the standard representation of $\mathrm{Sp}(1)$ on $\mathbf{H} \cong \mathrm{C}^{2}$. Hence $V \otimes V^{\prime}$ is of real type, therefore not admissible.

In case (v), the irreducible representation of $\mathrm{Sp}(2) \mathrm{Sp}(1)$ is $V \otimes V^{\prime}$, where $V$ is the fundamental representation of $\operatorname{Sp}(2)$ on $\mathbf{H}^{2}$, and $V^{\prime}$ is the 4-dimensional representation of $\mathrm{SU}(2) \cong \mathrm{Sp}(1)$ on the complex space of homogeneous polynomials in two variables and of degree three. Clearly, both $V$ and $V^{\prime}$ are quaternionic. This implies that $V \otimes V^{\prime}$ is real. Therefore we can write $V \otimes V^{\prime}=$ $\mathrm{U} \oplus \mathrm{U}$, where $\mathrm{U}$ is a real irreducible representation of $\mathrm{Sp}(2) \mathrm{Sp}(1)$ of real dimension sixteen. So $V \otimes V^{\prime}$ is admissible.

(7) $\operatorname{Spin}(7)$. There is only one complex 8-dimensional irreducible representation $\Delta^{3}$ of $\operatorname{Spin}(7)$ and no 16 -dimensional ones. The representation $\Delta^{3}$ is the only possibly admissible one for dimensional reasons. It is known that $\Delta^{3}$ is self-conjugate and of real type. Hence $\Delta^{3}$ is not admissible.

Now we see that the only admissible representation is $V \otimes V^{\prime}$ of $\operatorname{Sp}(2) \mathrm{Sp}(1)$, where $V$ is the fundamental representation of $\mathrm{Sp}(2)$ on $\mathrm{H}^{2}$ and $V^{\prime}$ can be seen as the standard action of $\mathrm{SU}(2)=\mathrm{Sp}(1)$ on the complex space of homogeneous polynomials in two variables and of degree three. Furthermore, $V \otimes V^{\prime}=\mathrm{U} \oplus \mathrm{U}$, where $\mathrm{U}$ is a real irreducible representation of real dimension sixteen with respect to some real structure. For different real structures, the decomposition $V \otimes V^{\prime}=\mathrm{U} \oplus \mathrm{U}$ gives rise to isomorphic $\mathrm{U}$ 's. Hence we need only to choose one real structure to see if $U$ gives rise to a homogeneous foliation.

Recall that $U$ can be taken as the eigenspace of the positive eigenvalue one of the real structures. On the other hand, if $J$ is a quaternionic structure of $V$ and $J^{\prime}$ is a quaternionic structure of $V^{\prime}$, then $J \otimes J^{\prime}$ is a real structure of $V \otimes V^{\prime}$. We choose $J$ for $V$ to be the multiplication by $j$ from left. Under the identification of $\mathbf{H}=\mathbf{C}+\mathbf{C}_{j}, J$ is composed of the $C$-linear map induced by right multiplication of $j$ followed by complex conjugation $c: \mathbf{C}^{4} \rightarrow$ $\mathbf{C}^{4}$ such that $c(w)=\bar{w}$. The right multiplication by $j$ can be written as $u+v j \mapsto(u+v j) j=-v+u j$. Hence for $z=\left(z_{1}, z_{2}, z_{3}, z_{4}\right) \in \mathbf{C}^{4}$, or $z=\left(z_{1}+z_{3} j, z_{2}+z_{4} j\right)$, we have

$$
J z=c(z j)=c\left(-z_{3}+z_{1} j,-z_{4}+z_{2} j\right)
$$

or

$$
J z=c\left(-z_{3},-z_{4}, z_{1}, z_{2}\right)=\left(-\bar{z}_{3},-\bar{z}_{4}, \bar{z}_{1}, \bar{z}_{2}\right) .
$$

To choose $J^{\prime}$ for $V^{\prime}$, we identify $V^{\prime}$ with the standard action of $\mathrm{SU}(2)$ on the complex space of homogeneous polynomials in two variables and of degree three. For any $g \in \mathrm{SU}(2)$, and any $f\left(z_{1}, z_{2}\right) \in V^{\prime}$, the action of $g$ is given by $\left(g, f\left(z_{1}, z_{2}\right)\right) \mapsto f\left(\left(z_{1}, z_{2}\right) g\right)$.

Precisely, if we choose the monomials $z_{1}^{3}, z_{1}^{2} z_{2}, z_{1} z_{2}^{2}$, and $z_{2}^{3}$ as the linear base of $V^{\prime}$, and write

$$
g=\left(\begin{array}{cc}
a & b \\
-\bar{b} & \bar{a}
\end{array}\right) \in \mathrm{SU}(2),
$$


then the action on $V^{\prime}$ is given by the matrix $A_{g}$

$$
A_{g}=\left(\begin{array}{cccc}
a^{3} & a^{2} b & a b^{2} & b^{3} \\
-3 a^{2} \bar{b} & a^{2} \bar{a}-2 a b \bar{b} & 2 a \bar{a} b-\bar{b} b^{2} & 3 b^{2} \bar{a} \\
3 a \bar{b}^{2} & b \bar{b}^{2}-2 a \bar{a} \bar{b} & a \bar{a}^{2}-2 \bar{a} b \bar{b} & 3 \bar{a}^{2} b \\
-\bar{b}^{3} & \bar{a} \bar{b}^{2} & -\bar{a}^{2} b & \bar{a}^{3}
\end{array}\right) .
$$

Let

$$
\omega=\left(\begin{array}{cccc}
0 & 0 & 0 & 1 \\
0 & 0 & -1 & 0 \\
0 & 1 & 0 & 0 \\
-1 & 0 & 0 & 0
\end{array}\right)
$$

and $J^{\prime}=c \omega$. It is clear that $c \omega=\omega c$ and $J^{\prime 2}=(c \omega)^{2}=\omega^{2}=-1$. By computation, $\omega A=\bar{A} \omega$ and $c A=\bar{A} c$, where $\bar{A}=A_{\bar{g}}$. So $A c \omega=c \bar{A} \omega=$ $c \omega A$, that is, $J^{\prime} A=A J^{\prime}$. Therefore $J^{\prime}$ is a quaternionic structure of $V^{\prime}$.

Since the eigenspace of the eigenvalue positive one of $J \otimes J^{\prime}$ is given by

$\left\{v \otimes v^{\prime} \oplus\left(J \otimes J^{\prime}\right)\left(v \otimes v^{\prime}\right)=v \otimes v^{\prime} \oplus(J v) \otimes\left(J^{\prime} v^{\prime}\right) \mid v \in V, v^{\prime} \in V^{\prime}\right\}$, and for $\left(u_{1}+v_{1} j, u_{2}+v_{2} j\right) \in \mathbf{H}^{2},\left(a_{1}, a_{2}, a_{3}, a_{4}\right) \in \mathbf{C}^{4}$, we have

$$
\begin{gathered}
J\left(u_{1}+v_{1} j, u_{2}+v_{2} j\right)=\left(-\bar{v}_{1}+\bar{u}_{1} j,-\bar{v}_{2}+\bar{u}_{2} j\right), \\
J^{\prime}\left(a_{1}, a_{2}, a_{3}, a_{4}\right)=\left(\bar{a}_{4},-\bar{a}_{3}, \bar{a}_{2},-\bar{a}_{1}\right) .
\end{gathered}
$$

We see that

$$
\begin{aligned}
U=\left\{\left(u_{1}+\right.\right. & \left.v_{1} j, u_{2}+v_{2} j\right) \otimes\left(a_{1}, a_{2}, a_{3}, a_{4}\right) \\
+ & \left(-\bar{v}_{1}+\bar{u}_{1} j,-\bar{v}_{2}+\bar{u}_{2} j\right) \otimes\left(\bar{a}_{4},-\bar{a}_{3}, \bar{a}_{2},-\bar{a}_{1}\right): \\
& \left.\left(u_{1}+v_{1} j, u_{2}+v_{2} j\right) \in \mathbf{H}^{2},\left(a_{1}, a_{2}, a_{3}, a_{4}\right) \in \mathbf{C}^{4}\right\} .
\end{aligned}
$$

We now look at a specific point $p: p=(1,0) \otimes(1,0,0,0)+(j, 0) \otimes$ $(0,0,0,-1)$, and compute the dimension of the isotropy group at $p$.

For any $g \in \mathrm{Sp}(2) \mathrm{Sp}(1), g$ can be written as

$$
g=\left(\left(\begin{array}{cc}
\alpha & \beta \\
\lambda & \delta
\end{array}\right),\left(\begin{array}{cc}
a & b \\
-\bar{b} & \bar{a}
\end{array}\right)\right)
$$

where

$$
\left(\begin{array}{ll}
\alpha & \beta \\
\lambda & \delta
\end{array}\right) \in \operatorname{Sp}(2),\left(\begin{array}{cc}
a & b \\
-\bar{b} & \bar{a}
\end{array}\right) \in \mathrm{SU}(2)=\operatorname{Sp}(1) .
$$

The image of $p$ under the action of $g$ is

$$
(\alpha, \gamma) \otimes\left(a^{3},-3 a^{2} \bar{b}, 3 a \bar{b}^{2},-\bar{b}^{3}\right)+(\alpha j, \gamma j) \otimes\left(-b^{3},-3 b^{2} \bar{a},-3 \bar{a}^{2} b,-\bar{a}^{3}\right) .
$$

If $g p=p$, then we see immediately that $\gamma=0$. Furthermore, by solving the equation $g p=p$, we have $a=0$ or $b=0$, and in both cases $\beta=0$. For $b=0$, we have $a \neq 0$ and

$$
g=\left(\left(\begin{array}{cc}
-1 & 0 \\
0 & \delta
\end{array}\right),\left(\begin{array}{cc}
a & 0 \\
0 & \bar{a}
\end{array}\right)\right), \quad|\delta|=1, \quad a^{6}=1 .
$$

For $a=0$, we have $b \neq 0$, and

$$
g=\left(\left(\begin{array}{cc}
-j & 0 \\
0 & \delta
\end{array}\right),\left(\begin{array}{cc}
0 & b \\
-\bar{b} & 0
\end{array}\right)\right), \quad|\delta|=1, \quad b^{6}=1
$$


Since all the elements of the form

$$
\left(\left(\begin{array}{cc}
-j & 0 \\
0 & \delta
\end{array}\right),\left(\begin{array}{cc}
0 & b \\
-\bar{b} & 0
\end{array}\right)\right)
$$

are conjugate to the elements of the isotropy group at $p$ of the form

$$
\left(\left(\begin{array}{cc}
-1 & 0 \\
0 & \delta
\end{array}\right),\left(\begin{array}{ll}
a & 0 \\
0 & \bar{a}
\end{array}\right)\right),
$$

it follows that the identity component of the isotropy group at $p$ is isomorphic to $S^{3}$. So we know that the dimension of the isotropy group at $p$ is equal to $\operatorname{dim}\left(S^{3}\right)=3$.

Note that $\operatorname{dim}(\operatorname{Sp}(2))=10$ and $\operatorname{dim}(\operatorname{Sp}(1))=3$, so the dimension of the orbit through $p$ has dimension ten, which shows that the orbit of the action through $p$ is not covered by $S^{7}$.

Thus Step III is completed and so is the proof of the Main Theorem 1.2.

\section{ACKNOWLEDGMENT}

The author would like to thank his thesis advisor Dr. Wolfgang Ziller for the support, guidance, and encouragement during the time this work was done.

\section{REFERENCES}

1. A. Besse, Einstein manifolds, Springer-Verlag, New York, 1985.

2. T. Brocker and T. tom Dieck, Representations of compact Lie groups, Springer-Verlag, New York, 1985.

3. W. Browder, Higher torsion in H-spaces, Trans. Amer. Math. Soc. 108 (1963), 353-375.

4. E. Ghys, Feuilletages Riemanniens sur les varietes simplement connexes, Ann. Inst. Fourier (Grenoble) 34 (1984), 203-223.

5. D. Gromoll and K. Grove, A generalization of Berger's rigidity theorem for positively curved manifolds, Ann. Sci. École Norm. Sup. (4) 20 (1987), 227-239.

6. __ One-dimensional metric foliations in constant curvature spaces, Differential Geometry and Complex Analysis, H.E. Rauch Memorial Volume, Springer-Verlag, New York, 1985, pp. 165-168.

7. _ The low dimensional metric foliations of Euclidean spheres, J. Differential Geom. 28 (1988), 143-156.

8. A. Haefliger, Groupoids d'holonomie et classifiants, Asterisque 116 (1984), 70-97.

Department of Mathematics, University of Pennsylvania, Philadelphia, PennsylvaNIA, 19104

Current address: Web Development Corporation, 415 McFarlan Road, Suite 100, Kennett Square, Pennsylvania 19348

E-mail address: lu@web.com 